\title{
Isowarp and Conwarp: Warps that Exactly Comply with Weak-Perspective Projection of Deforming Objects
}

\author{
Daniel Pizarro \\ dani.pizarro@gmail.com \\ Adrien Bartoli \\ adrien.bartoli@gmail.com \\ Toby Collins \\ toby.collins@gmail.com
}

ISIT-UMR 6284 CNRS

Universite d'Auvergne

Clermont Ferrand, France

\begin{abstract}
This paper studies the problem of surface reconstruction from a single image, given a template of the surface. We investigate the variational structure of the reconstruction problem for isometric and conformal deformations and weak-perspective camera projections. We give analytical solutions for the surface shape given that the registration warp between the template and the input image meets specific differential constraints. We explore those constraints, giving an optimization strategy to compute warps that comply with isometric and conformal deformations under weak-perspective projection. We study the performance of the proposed algorithms with synthetic and real datasets. The experiments show that surface reconstruction with weak-perspective is accurate when using cameras with large focal lengths and improves state of the art methods.
\end{abstract}

\section{Introduction}

Deformable surface reconstruction from monocular views has been intensively studied during the last few years $[2,10]$. In the template-based case, the goal is to obtain the 3D shape of the deformed template from a single image. This problem is ill-posed without constraints on how the surface deforms. Template-based surface reconstruction involves two problems: $i$ ) deformable image registration (e.g. from point correspondences, contours or image texture). ii) surface $3 \mathrm{D}$ shape inference from deformation constraints and the camera projection.

Image matching and registration of deformable surfaces counts with several contributions $[8,9,10]$ that make this process highly automatic and robust. Shape inference methods have been proposed for a variety of constraints: low-rank shape priors [5, 10], temporal deformation smoothness [11], isometric deformations [2, 3, 7] and conformal deformations [2]. Isometric and conformal constraints accurately model deformations in many real cases and they are described with simple differential constraints. Isometric reconstruction from perspective camera views has attracted much of the attention. Early approaches relax the nonconvex isometric constraints to inextensibility with the so-called maximum depth heuristic $[7,10]$. The idea is to maximize point depths so that the Euclidean distance between 
every pair of points is upper bounded by its geodesic distance, computed in the template. This problem is convex and, although a relaxation of the original problem, gives accurate reconstructions. Very recently [2] provides analytical solutions for isometric and conformal deformations by posing it as a system of Partial Differential Equations (PDEs).

We study surface reconstruction with isometric and conformal deformations and weakperspective projection. There are few works studying the reconstruction problem for weakperspective $[4,13]$ and orthographic projections [6]. In affine projection conditions the maximum depth heuristic [7, 10] and current analytical solutions [2] do not apply. In real scenarios, cameras with large focal lengths and shallow scene depth produce images close to affine projection conditions. This paper gives analytical solutions and shows that the registration between the template and the input image is not independent of the reconstruction. We define registration warps that comply with the weak-perspective projection of isometric and conformal surfaces, ensuring analytical solutions to compute surface's shape. We show that this method is more accurate than state-of-art methods based on perspective models, when the camera conditions are close to affine. In $\$ 2$ we present the definitions and the mathematical modelling of the reconstruction problem in form of PDE. In $\S 3$ and $\S 4$ we present the analytical solutions for isometric and conformal deformations. In $\$ 5$ we give insight about the reconstruction algorithm based on the solutions. We present the experimental results in $\S 6$.

\section{Mathematical Modeling}

Figure (1) shows the different functional relationships between the template and the deformed surface. The template is represented as a surface $\mathcal{T} \subset \mathbb{R}^{3}$ embedded in $3 \mathrm{D}$. We assume that $\mathcal{T}$ has disk topology, admitting a 2D parameterization with conformal flattening [12]. The flat template is denoted as $\mathcal{P} \subset \mathbb{R}^{2}$. We denote the flattening as the invertible mapping function $\Delta \subset \mathbb{C}_{1}$.

The input image $\mathcal{I}$ shows the projection of the deformed template $\mathcal{S} \subset \mathbb{R}^{3}$. The camera model $\Pi$ is a weak-perspective projection. A point $\mathbf{Q} \in \mathcal{S}$ is projected on the point $\mathbf{p} \in \mathcal{I}$ according to the following linear equation:

$$
\mathbf{q}=\Pi(\mathbf{Q})=s\left(\mathbf{I}_{2 \times 2} \mathbf{0}\right) \mathbf{Q}=s\left(Q_{x} Q_{y}\right)^{\top},
$$

where $s=\frac{1}{d}$ and $d_{z}$ is the average scene depth. Considering $s=1$ implies an orthographic camera model. We assume that the camera is calibrated and the image coordinates $\mathbf{q}$ are normalized (i.e. $\mathbf{q}=A^{-1} \hat{\mathbf{q}}$, with $A$ the intrinsic camera matrix and $\hat{\mathbf{q}}$ expressed in pixels). We define $\eta \in \mathbb{C}_{1}$ as the warp mapping a point $\mathbf{p} \in \mathcal{P}$ in the flattened template to a corresponding point $\mathbf{q}=\eta(\mathbf{p}) \in \mathcal{I}$ in the image. Function $\psi \in \mathbb{C}_{1}$ models deformations between the template $\mathcal{T}$ and the deformed surface $\mathcal{S}$. Conformal and isometric deformations are described with conditions on the first derivatives of $\psi$, namely $J_{\psi}$ :

$$
J_{\psi}^{\top} J_{\psi}=\mu \mathbf{I}_{3 \times 3}, \quad \text { where } \quad \mu= \begin{cases}1 & \text { Isometric } \\ \mu \in \mathbb{C}_{1} \text { unknown } & \text { Conformal. }\end{cases}
$$

We define $\varphi=(\psi \circ \Delta) \in \mathbb{C}_{1}$ which maps points $\mathbf{p} \in \mathcal{P}$ in the $2 \mathrm{D}$ template to points $Q=$ $\varphi(\mathbf{p}) \in \mathcal{S}$ in the deformed surface. Using the properties of the conformal flattening and the 
conditions (2), the following differential properties are derived:

$$
J_{\varphi}^{\top} J_{\varphi}=\lambda \mathbf{I}_{2 \times 2}, \quad \text { where } \quad \lambda= \begin{cases}\sqrt{\operatorname{det}\left(J_{\Delta}^{\top} J_{\Delta}\right)} & \text { Isometric } \\ \lambda \in \mathbb{C}_{1} \text { unknown } & \text { Conformal. }\end{cases}
$$

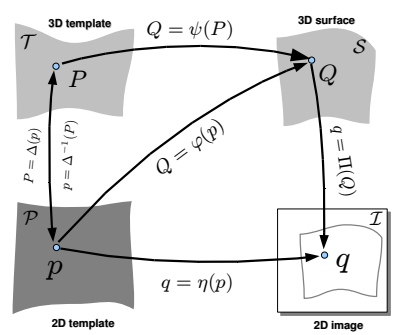

Figure 1: General model of template-based 3D reconstruction

\section{Reconstruction with Isometric Deformation}

\subsection{Differential Formulation}

Surface reconstruction under isometric deformation constraints can be posed as:

$$
\text { Find } \varphi \in \mathbb{C}_{1} s \in \mathbb{R} \quad \text { s.t. }\left\{\begin{array}{lr}
\eta=\Pi \circ \varphi \quad \text { Reprojection constraint } \\
J_{\varphi}^{\top} J_{\varphi}=\lambda \mathbf{I}_{2 \times 2} & \text { Isometry constraint, }
\end{array}\right.
$$

where function $\lambda$ is given by equation (3) and the warp $\eta$ is obtained from the registration between the input image and the flattened template. We show in this section that problem (4) can be expressed as a nonlinear system of PDEs.

We first define $\eta=\left(\eta_{x}, \eta_{y}\right)^{\top}$ and $\varphi=\left(\varphi_{x}, \varphi_{y}, \varphi_{z}\right)^{\top}$ by their scalar components. Following the projection constraint we have:

$$
\eta=s\left(\varphi_{x}, \varphi_{y}\right)^{\top} \Rightarrow \eta_{x}=s \varphi_{x} \quad \eta_{y}=s \varphi_{y}
$$

The isometric deformation constraint is rewritten as:

$$
J_{\varphi}^{\top} J_{\varphi}=\lambda \mathbf{I}_{2 \times 2} \quad J_{\varphi}=\left(\begin{array}{c}
\frac{1}{s} \frac{\partial \eta}{\partial x} \frac{1}{s} \frac{\partial \eta}{\partial y} \\
\frac{\partial \varphi_{z}}{\partial x} \frac{\partial \varphi_{z}}{\partial y}
\end{array}\right) .
$$

From equation (6), the following nonlinear system of PDEs is derived:

$$
\left\{\begin{array}{l}
\chi_{x}+s^{2}\left(\frac{\partial \varphi_{z}}{\partial x}\right)^{2}=\chi_{y}+s^{2}\left(\frac{\partial \varphi_{z}}{\partial y}\right)^{2}=\lambda s^{2} \\
\xi+s^{2}\left(\frac{\partial \varphi_{z}}{\partial x}\right)\left(\frac{\partial \varphi_{z}}{\partial y}\right)=0
\end{array}\right.
$$


where:

$$
\chi_{x}=\frac{\partial \eta^{\top}}{\partial x} \frac{\partial \eta}{\partial x} \quad \chi_{y}=\frac{\partial \eta^{\top}}{\partial y} \frac{\partial \eta}{\partial y} \quad \xi=\frac{\partial \eta^{\top}}{\partial x} \frac{\partial \eta}{\partial y} .
$$

Finding the solution of system (7) in terms of the derivatives of $\varphi_{z}$ and $s$ allows us to reconstruct all components of $\varphi$ up to an additive constant in $\varphi_{z}$.

\subsection{Analytical Solution}

The derivatives of $\varphi_{z}$ are given by the first two equations of (7):

$$
\Gamma_{x}=\frac{\partial \varphi_{z}}{\partial x}=\delta_{1} \sqrt{s^{2} \lambda-\chi_{x}} \quad \Gamma_{y}=\frac{\partial \varphi_{z}}{\partial y}=\delta_{2} \sqrt{s^{2} \lambda-\chi_{y}},
$$

where $\delta_{1}$ and $\delta_{2}$ are piecewise constant functions maping to $\{-1 ; 1\}$. By substitution of equalities (9) in the last equation of system (7) we find the following condition involving $\eta$, $s$ and functions $\delta_{1}$ and $\delta_{2}$ :

$$
\xi=\delta_{1} \delta_{2} \sqrt{s^{2} \lambda-\chi_{x}} \sqrt{s^{2} \lambda-\chi_{y}} \quad \delta_{1} \delta_{2}=\operatorname{sign}(\xi)
$$

As $\varphi_{z} \in \mathbb{C}_{1}$, each region of the template enclosed by the zero-level set of $\xi$ gives 2 possible solutions to the pair $\left(\delta_{1}, \delta_{2}\right)$ and thus to $\Gamma_{x}$ and $\Gamma_{y}$. By squaring terms in (10) we get the following differential condition in $\eta$ and $s$

$$
\left(s^{2} \lambda-\chi_{x}\right)\left(s^{2} \lambda-\chi_{y}\right)-\xi^{2}=0 .
$$

The value of the scalar $s$ can be obtained from (11), evaluated at any point $\mathbf{p} \in \mathcal{P}$. It results in a second order polynomial in $s^{2}$ with two solutions. We select the solution $s^{2}$ that guarantees that $\Gamma_{x}$ and $\Gamma_{y}$ are real numbers. It always corresponds to the following:

$$
s^{2}=\frac{1}{2 \lambda(\mathbf{p})}\left(\chi_{x}(\mathbf{p})+\chi_{y}(\mathbf{p})+\sqrt{\left(\chi_{x}(\mathbf{p})-\chi_{y}(\mathbf{p})\right)^{2}+\xi(\mathbf{p})^{2}}\right)
$$

We can then obtain $\varphi_{z}$ by integration of $\Gamma_{x}$ or $\Gamma_{y}$ (up to an unknown constant). The system (7) is thus redundant when $\eta$ complies exactly with (11).

\subsection{The Isowarp $\eta$}

When $\eta$ contains errors, the solutions of equations (9) and (12) do not guarantee neither that $\Gamma_{x}$ and $\Gamma_{y}$ share a primitive nor that they are exact solutions of system (7). In fact, with errors in $\eta$, equation (12) varies in function of $\mathbf{p}$, not being a constant scalar for all points in $\mathcal{P}$.

We define the isowarp as a function $\eta$ that represents the weak-perspective projection of an isometric deforming surface. If $\eta$ is an isowarp, system (7) has an exact solution and directly solves the isometric reconstruction problem (4). An isowarp $\eta$ satisfies the following system of PDEs:

$$
\begin{array}{r}
\left(s^{2} \lambda-\chi_{x}\left(s^{2} \lambda-\chi_{y}\right)-\xi^{2}=0\right. \\
\left(s^{2} \lambda-\chi_{y}\right)\left(s^{2} \frac{\partial \lambda}{\partial y}-\frac{\partial \chi_{x}}{\partial y}\right)^{2}-\left(s^{2} \lambda-\chi_{x}\right)\left(s^{2} \frac{\partial \lambda}{\partial x}-\frac{\partial \chi_{y}}{\partial x}\right)^{2}=0
\end{array}
$$


where $s \in \mathbb{R}^{+}$. Equation (13) ensures that system (7) has solutions with $s$ constant for any $\mathbf{p} \in \mathcal{P}$. Condition (13) ensures that $\Gamma_{x}$ and $\Gamma_{y}$ share a primitive by imposing $\frac{\partial \Gamma_{x}}{\partial y}=\frac{\partial \Gamma_{y}}{\partial x}$. Conditions (13) and (13) can be plugged into the registration problem as optimization constraints, forcing $\eta$ to be an isowarp.

\section{Reconstruction with Conformal Deformations}

\subsection{Differential Formulation}

Surface reconstruction under conformal deformation constraints can be posed as:

$$
\text { Find } \varphi \in \mathbb{C}_{1} \lambda \in \mathbb{C}_{1} s \in \mathbb{R} \quad \text { s.t. } \begin{cases}\eta=\Pi \circ \varphi & \text { Rerojection constraint } \\ J_{\varphi}^{\top} J_{\varphi}=\lambda \mathbf{I}_{2 \times 2} & \text { Isometric constraint, }\end{cases}
$$

Problem (14) is expressed as the same system of PDEs described in (7) for isometric deformations, with $\lambda$ an unknown function.

In the conformal case we find the following solutions to (7):

$$
\left\{\begin{array}{l}
\Gamma_{x}=\delta_{1} \delta_{2} \sqrt{s^{2} \lambda-\chi_{x}} \quad \Gamma_{y}=\delta_{1} \delta_{2} \sqrt{s^{2} \lambda-\chi_{y}} \quad \text { with } \quad \delta_{1} \delta_{2}=\operatorname{sign}(\xi) \\
\lambda=\frac{1}{2 s^{2}}\left(\chi_{x}+\chi_{y}+\sqrt{\left(\chi_{x}-\chi_{y}\right)^{2}+\xi^{2}}\right)
\end{array}\right.
$$

where we highlight that $s^{2}$ cancels by substitution of $\lambda$ in $\Gamma_{x}$ and $\Gamma_{y}$. The solution to $\varphi$ is thus defined up to a scale factor for conformal deformations and up to an additive constant in $\varphi_{z}$ due to integration. In addition, as in the isometric case, for each region where the sign of $\xi$ changes we obtain multiple solutions for $\Gamma_{x}$ and $\Gamma_{y}$.

\subsection{Conwarp}

In the conformal case the fact that $\lambda$ is an unknown function removes the first constraint (11) on $\eta$ we found for isometric deformations. Even with errors in $\eta$ we can always find $\Gamma_{x}$ and $\Gamma_{y}$ that are exact solutions of all equations in (7). However, we still need to ensure they share a primitive $\varphi_{z}$.

We define the conwarp as a function where the integrability condition of $\frac{\partial \Gamma_{x}}{\partial x}=\frac{\partial \Gamma_{y}}{\partial y}$ is met. A conwarps satisfies the following PDE:

$$
\left(s^{2} \lambda-\chi_{y}\right)\left(s^{2} \frac{\partial \lambda}{\partial y}-\frac{\partial \chi_{x}}{\partial y}\right)^{2}-\left(s^{2} \lambda-\chi_{x}\right)\left(s^{2} \frac{\partial \lambda}{\partial x}-\frac{\partial \chi_{y}}{\partial x}\right)^{2}=0,
$$

where $\lambda$ is given by (15).

\section{Reconstruction Algorithm}

This sections gives insights about how to convert the analytical solutions obtained in the last section into algorithms. Whether it is conformal or isometric reconstruction the main algorithm has two steps: 1 ) Compute $\eta$ (and additionally $s$ for isometric deformations) using 
image information (e.g. feature matching) and the constraints described in equations (13) and (13). 2) Compute $\varphi_{z}$ by numerical integration of $\Gamma_{x}$ and $\Gamma_{y}$, obtained from equations (9) and (15) for the isometric and conformal deformation models respectively.

\subsection{Computing Isowarps and Conwarps}

The following constrained optimization problem is proposed to find $\eta$ and $s$ :

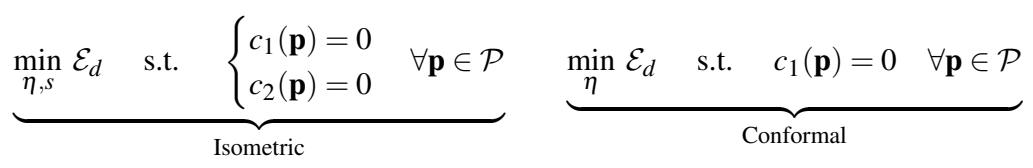

where $\mathcal{E}_{d}$ is a data functional measuring registration error. In case of feature-based methods $\mathcal{E}_{d}$ is usually the mean transport error between features in the template and input image. The set of constraints $c_{1}(\mathbf{p})$ and $c_{2}(\mathbf{p})$ correspond to equations (13) and (13) respectively at point $\mathbf{p} \in \mathcal{P}$. Problem (17) is non-convex. We propose a Lagrangian relaxation to include constraints and we use unconstrained nonlinear optimization (i.e. Levenberg-Marquardt) to reach the minimum:

$$
\min _{\eta, \mu}\left(\mathcal{E}_{d}+\sum_{j=1}^{M}\left(\gamma_{1} c_{1}^{2}\left(\mathbf{p}_{j}\right)+\gamma_{2} c_{2}^{2}\left(\mathbf{p}_{j}\right)\right)+\gamma_{s} \mathcal{E}_{s}\right)
$$

where $\{\mathbf{p}\}_{j=1}^{M}$ is a discrete uniform grid of $\mathcal{P} . \gamma_{1}$ and $\gamma_{2}$ are hyper-parameters that have to be tuned empirically. The functional $\mathcal{E}_{s}$ helps to find a smooth $\eta$ in areas of $\mathcal{P}$ where the solution is not constrained enough (e.g. at the boundaries of a surface without features). We use the bending energy [1] to impose smoothness.

To get an accurate initial guess for $\eta$, we remove the $c_{1}$ and $c_{2}$ functionals, solving the remaining convex problem. In the case of isometric deformations we initialize $s$ by using the median of the set $\left\{s_{j}\right\}_{j}^{M}$, where:

$$
s_{j}=\sqrt{\frac{1}{2 \lambda\left(\mathbf{p}_{j}\right)}\left(\chi_{x}\left(\mathbf{p}_{j}\right)+\chi_{y}\left(\mathbf{p}_{j}\right)+\sqrt{\left(\chi_{x}\left(\mathbf{p}_{j}\right)-\chi_{y}(\mathbf{p})\right)^{2}+\xi\left(\mathbf{p}_{j}\right)^{2}}\right)}
$$

\subsection{Computing $\varphi$}

As it is clear from the previous section, the solution to $\varphi$ is not unique neither for isometric nor conformal deformations. The number of solutions for $\Gamma_{x}$ and $\Gamma_{y}$ is given by the amount of regions of $\mathcal{P}$ where the sign of $\xi$ changes. For $N_{r}$ regions we have $2^{N_{r}}$ solutions for $\Gamma_{x}$ and $\Gamma_{y}$. For each of these solutions, $\varphi_{z}$ is obtained by integration up to a depth ambiguity $\varphi_{z}+z_{c}$. If the camera projection is very close to orthographic, more information is needed to resolve the ambiguity, such as shading [13] or temporal smoothing [4]. However, if the projection is perspective we know from [2] that we have a single solution for isometric deformations and a discrete set of them for conformal deformations (up to scale). In this paper we propose a simple strategy to select a single solution when the projection is not exactly orthographic. For each solution $\varphi$ we obtain the value of $z_{c}$ that minimizes the following cost:

$$
\varepsilon_{c}^{2}=\sum_{j=1}^{N}\left(\frac{\varphi_{x}\left(\mathbf{p}_{j}\right)}{\varphi_{z}\left(\mathbf{p}_{j}\right)+z_{c}}-x_{j}\right)^{2}+\left(\frac{\varphi_{y}\left(\mathbf{p}_{j}\right)}{\varphi_{z}\left(\mathbf{p}_{j}\right)+z_{c}}-y_{j}\right)^{2} .
$$


Synthetic generated images for $f=500 p x$

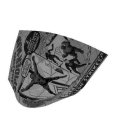

(a) Normal error vs $f$

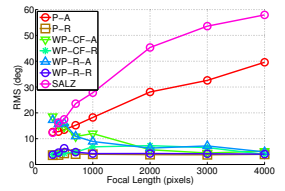

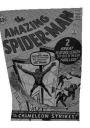

(b) Depth error vs $f$

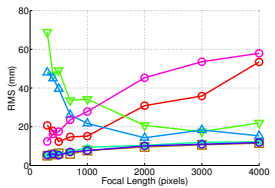

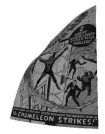

Experiments

Figure 2: Synthetic generated images and experimental results

(c) Normal error vs $\sigma$

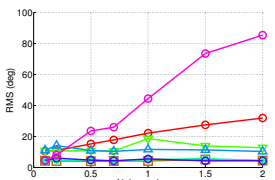

(d) Depth error vs $\sigma$

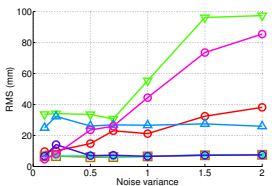

We finally keep the solution with the smallest $\varepsilon_{c}^{2}$.

\section{Experimental Validation}

The name of algorithms that we evaluate with datasets are encoded with the following acronyms: P-A is the perspective, analytical reconstruction [2]. P-R is P-A followed by nonlinear refinement [3]. WP-CF-A is the weak-perspective model using closed form solution for $\eta$ (removing $c_{1}$ and $c_{2}$ ) and then obtaining $\varphi$ directly using closed-form integration. WP-CF-R is WP-CF-A followed by nonlinear refinement. WP-R-A uses non-linear refinement to get an isowarp (or conwarp ) $\eta$. WP-R-R is WP-R-A followed by nonlinear refinement. Finally $\mathbf{S A L Z}$ is isometric reconstruction based on the maximum depth heuristic [10].

\subsection{Synthetic dataset}

We use ruled surfaces to simulate isometric deformations of a flat template of size $640 \mathrm{~mm} \times$ $480 \mathrm{~mm}$. We generate synthetic images using a pin-hole camera with varying focal lengths $f \in[400,500,700,800,1000,2000,3000,4000]$ px. On top of figure 2 we show 5 deformations used for the experiments. We randomly generate sets of $N=100$ point correspondences between the template and the images. We use two performance indicators: $\varepsilon$ is the error (in $\mathrm{mm}$ ) between 3D points in the ground-truth surface and their correspondences in reconstructed shape. $\varepsilon_{n}$ is the error (in degrees) between the ground-truth normals and the normals recovered with the model in a regular grid of $100 \times 100$ points. To compute $\varepsilon_{n}$ for WP-CF-A and WP-R-A we select the normal that is closest to the ground-truth so that we remove the effect of selecting the wrong solution which does appear in $\varepsilon$. We test the algorithm against varying focal length $f$ (see figure 2.(a) and 2.(b) ) and noise variance $\sigma$ (figure 2.(c) and 2.(d)). The defaults are $f=1000 \mathrm{px}$ and $\sigma=0.5 \mathrm{px}$. We can see from figure 2 that the WP analytical solutions improve P-A and SALZ when $f$ is large. We can also conclude from figure 2.(c) and 2.(d) that WP-CF-A is more unstable for recovering the correct shape when $\sigma$ increases, even if $\varepsilon_{n}$ is accurate. The solution based on isowarps is the most stable and accurate given all indicators. 


\subsection{Real Data}

In this section we propose experiments to test the proposed methods on real surfaces. For the isometric case we select a bending sheet of paper. In the conformal case we use a balloon with two levels of air volume inside. The inflation of the balloon produce deformations that approximate conformal deformations. In all the experiments we use a commercial DSLR camera with variable zoom optics. Image resolution is $2304 \times 3456$ px.

Bending paper dataset. The bending paper dataset is composed of two basic shapes (shape 1 and 2) projected with different viewpoints and 4 different focal lengths. The ground truth surface of the paper in camera reference is obtained using commercial Structure-fromMotion software. We show results with shape 1 and $f=12166 \mathrm{px}$ in figures 3.(a-h) and with shape 2 and $f=7924$ px in figures 3.(i-p). We visualize $\eta$ as a mesh overlaid onto the input images in figures 3.(a,c,i,k) for smooth and isowarp (WP-R) constraints. In figures 3.(b,d,j,l) we show the flat template overlaid with the different regions where the sign of $\xi$ changes. The shape obtained with the different methods is shown in figures 3.(f,g,h) and 3.(n,o,p). Finally in the bottom part of figure 3 we show a table with the depth error $\varepsilon$ and normal error $\varepsilon_{n}$ for all input images used in the experiment, including those detailed in the top part of figure 3 (i.e. shaded cells). It is interesting to see that methods based on isowarps obtain accurate results on most of the cases and focal lengths. In many cases WP-R-A reaches a solution near the same minimum obtained after refinement. The methods based on smooth warps (WP-CF) fail to find the correct solution in most of the cases. As we expected, the perspective method $\mathrm{P}-\mathrm{A}$ is accurate with short-focal lengths. For bigger $f$ we found for one case that the P-R solution is stuck in a local minimum while WP-R-R method is able to get a better solution.

Balloon dataset . The balloon dataset shows the performance of the proposed method with conformal deformations. The template is obtained using a commercial SfM software. The flat template is obtained using conformal flattening. In the conformal case the solution is obtained up to a scale ambiguity and a discrete amount of solutions, both in the WP and P cases. We obtain the solution and the scale that best fit the ground-truth data. We provide depth error $\varepsilon$ for all methods. Last row of figure 4 clearly shows that weak perspective methods are very accurate. In this case WP-CF and WP-R methods show the same behavior which means that conwarps and smooth warps are similar in this dataset.

\section{Conclusions}

This paper proposed analytical solutions for the template-based reconstruction of surfaces from weak-perspective monocular views. We showed that the weak-perspective model is especially useful for cameras with large focal lengths. We developed the tools and the theoretical background to obtain surface deformations for isometric and conformal surfaces. We also developed conditions on the registration warp so that it complies with the reconstruction problem, defining the isowarp and the conwarp. The experiments demonstrate that the algorithms developed in this paper give accurate and stable results with real images under several conditions of focal length, deformations and noise. 


\section{Shape $1 \quad f=12166 \mathrm{px}$}

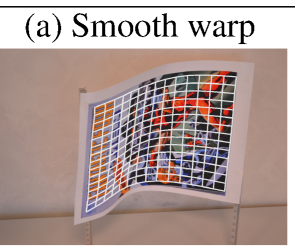

(e) Template (b) WP-CF regions

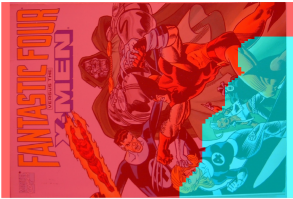

(f) WP-CF 3D shape

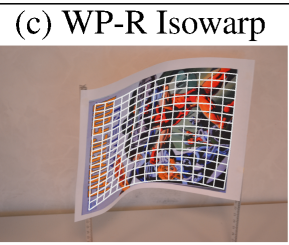

(g) WP-R 3D shape (d) WP-R regions

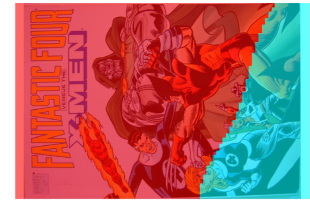

(h) Other methods
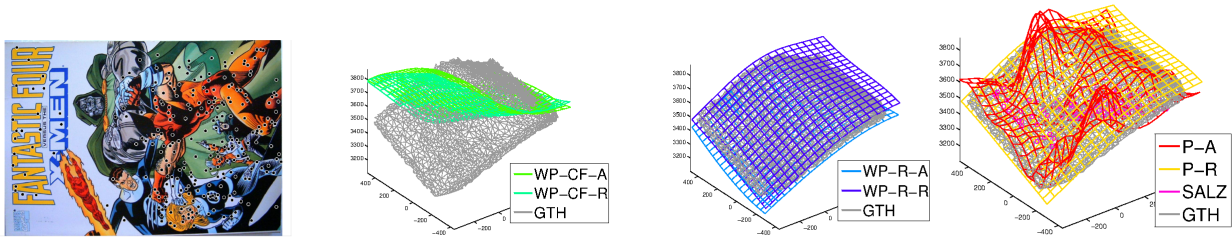

Shape $2 \quad f=7942 \mathrm{px}$

(i) Smooth warp

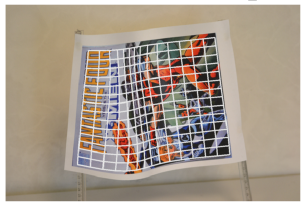

(m) Template

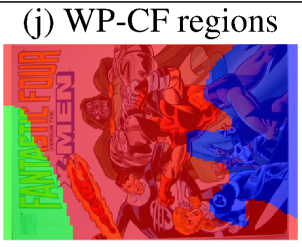

(n) WP-CF 3D shape

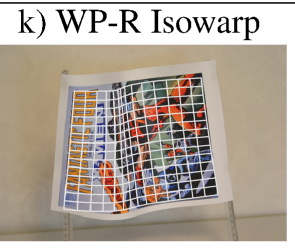

(o) WP-R 3D shape

(1) WP-R regions

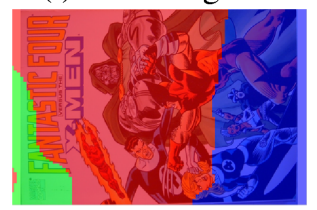

(p) Other methods

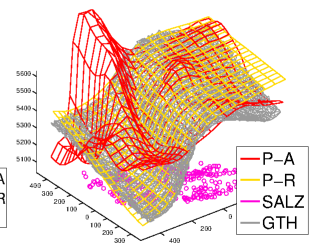

Shape 2

Focal Length

Shape 1

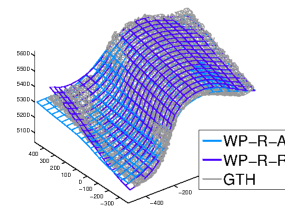

\begin{tabular}{l|c|c|c|} 
P-R-R & P-A & P-R & SALZ \\
\hline 8.49 & 16.11 & 8.47 & 63.36 \\
8.16 & 34.19 & 8.16 & 28.27 \\
\hline 6.89 & 11.15 & 10.67 & 70.57 \\
8.69 & 29.47 & 18.69 & 30.20 \\
\hline 0.74 & 29.83 & 10.61 & 62.31 \\
5.65 & 37.38 & 5.65 & 21.73 \\
\hline 5.26 & 39.10 & 11.32 & 123.80 \\
0.21 & 40.90 & 10.21 & 42.16 \\
\hline
\end{tabular}

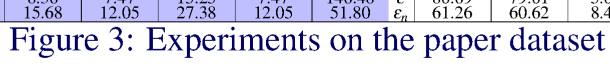

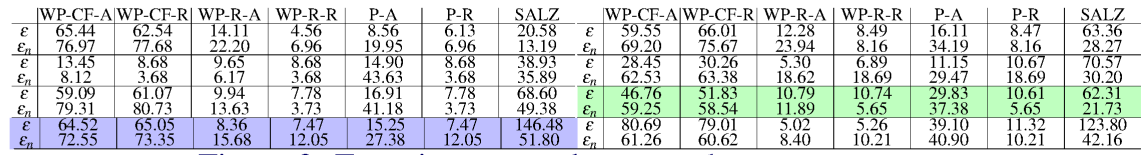

\begin{tabular}{|c|c|c|c|c|c|c|}
\hline Template & \multicolumn{2}{|c|}{ Input $\mathrm{f}=3913 \mathrm{px}$} & \multicolumn{3}{|c|}{ Input $\mathrm{f}=3613 \mathrm{px}$} & \\
\hline Focal (px)/RMS (mm) & $\mathrm{P}-\mathrm{A}$ & P-R & WP-CF-A & WP-CF-R & WP-R-A & WP-R-R \\
\hline 7666.8 & 2.88 & 2.23 & 2.088 & 2.072 & 2.088 & 2.072 \\
\hline 5673.5 & 2.41 & 1.53 & 4.26 & 2.47 & 4.26 & 2.47 \\
\hline 3613.9 & 3.23 & 1.37 & 5.83 & 4.10 & 5.83 & 4.10 \\
\hline
\end{tabular}

Figure 4: Experiments with the balloon dataset 


\section{Acknowledgements}

This research has received funding from the EU's FP7 through the ERC research grant 307483 FLEXABLE.

\section{References}

[1] A. Bartoli. Maximizing the predictivity of smooth deformable image warps through cross-validation. Journal of Mathematical Imaging and Vision, 31(2):133-145, 2008.

[2] A. Bartoli, Y. Gérard, F. Chadebecq, and T. Collins. On template-based reconstruction from a single view: Analytical solutions and proofs of well-posedness for developable, isometric and conformal surfaces. In Computer Vision and Pattern Recognition (CVPR), 2012 IEEE Conference on, pages 2026-2033. IEEE, 2012.

[3] F. Brunet, R. Hartley, A. Bartoli, N. Navab, and R. Malgouyres. Monocular templatebased reconstruction of smooth and inextensible surfaces. Computer Vision-ACCV 2010, pages 52-66, 2011.

[4] T Collins and A Bartoli. Locally affine and planar deformable surface reconstruction from video. In International Workshop on Vision, Modeling and Visualization, pages 339-346, 2010.

[5] A. Del Bue, X. Llad, and L. Agapito. Non-rigid metric shape and motion recovery from uncalibrated images using priors. In Computer Vision and Pattern Recognition, 2006 IEEE Computer Society Conference on, volume 1, pages 1191-1198. IEEE, 2006.

[6] Nail A Gumerov, Ali Zandifar, Ramani Duraiswami, and Larry S Davis. 3d structure recovery and unwarping of surfaces applicable to planes. International journal of computer vision, 66(3):261-281, 2006.

[7] M. Perriollat, R. Hartley, and A. Bartoli. Monocular template-based reconstruction of inextensible surfaces. International journal of computer vision, 95(2):124-137, 2011.

[8] J. Pilet, V. Lepetit, and P. Fua. Fast non-rigid surface detection, registration and realistic augmentation. International Journal of Computer Vision, 76(2):109-122, 2008.

[9] D. Pizarro and A. Bartoli. Feature-based deformable surface detection with selfocclusion reasoning. International journal of computer vision, 97(1):54-70, 2012.

[10] M. Salzmann and P. Fua. Linear local models for monocular reconstruction of deformable surfaces. Pattern Analysis and Machine Intelligence, IEEE Transactions on, 33(5):931-944, 2011.

[11] M. Salzmann, R. Hartley, and P. Fua. Convex optimization for deformable surface 3-d tracking. In Computer Vision, 2007. ICCV 2007. IEEE 11th International Conference on, pages 1-8. IEEE, 2007.

[12] A. Sheffer, B. Lévy, M. Mogilnitsky, and A. Bogomyakov. Abf++: fast and robust angle based flattening. ACM Transactions on Graphics (TOG), 24(2):311-330, 2005. 
[13] Ryan White and David A Forsyth. Combining cues: Shape from shading and texture. In Computer Vision and Pattern Recognition, 2006 IEEE Computer Society Conference on, volume 2, pages 1809-1816. IEEE, 2006. 